\title{
Irreversibility and Second Law ${ }^{1)}$
}

W. Muschik

Institut für Theoretische Physik, Technische Universität Berlin, Berlin, Germany

Registration Number 770

\begin{abstract}
According to M. Eigen there are two reasons for irreversibility: weak and strict temporality. Measures for irreversibility are defined by different formulations of the 2nd law which can be divided into two classes: the more general in time integrated formulations as Clausius' inequality and the more special in time local formulations as the non-negativity of the entropy production density. The logical relations between eight different formulations of the $2^{\text {nd }}$ law are investigated and discussed.
\end{abstract}

\section{Introduction}

There is no doubt about it, that there are phenomena which are irreversible: A flower is fading away, a glass breaks, a man becomes old. We now consider a box which is divided into two parts by a slide. One of these two parts contains a gas, the other part is evacuated. If now the slide is removed, the gas is diffusing into the vacuum, so that after a certain time the gas is distributed equally in both the parts of the box, apart from some fluctuations. If we take a movie of this diffusion into vacuum, and if we present it backward to an audience, they see the equally distributed gas going without any reason into one of the box's part and that finally the slide is moving in dividing the box into two parts, one of them just containing the gas and the other vacuum. Each person of the audience will affirm, that she or he never before saw such a phenomenon in nature, and that she or he supposes that by mistake the movie was presented backward.

This simple example demonstrates that we all have some experience, if a phenomenon can be observed in nature or if it is impossible to watch it. But this rough feeling with respect to existence or non-existence of a phenomenon demands a more specific analytical expression which is given by one of the various formulations of the second law [1]. The second law of thermodynamics has received so many formulation, not all equivalent to each other, that we can say that there are as many "second laws" as there

1) Partially delivered at the $2^{\text {nd }}$ Workshop on Dissipation in Physical Systems, Sep. 1-3, 1997 in Borków, Poland 
are authors [2]. But before discussing some of these formulations'we have to specify the concept of irreversibility.

First of all we have to distinguish between the original phenomenon and its backward presented movie: Henceforth the original phenomenon is denoted as a process, whereas its backward presented movie is called the reversed process. Furthermore we distinguish between possible and impossible processes. "Possible" means, that such a process can be observed in nature, whereas "impossible" means, that up to now such a process was not observed, and that we suppose that this process is not existent in nature. By these concepts "possible" and "impossible" we find the observer's experience of nature, as we have discussed it in the simple example above. Equipped with these concepts we can give a definition of "irreversible".

Definition: A possible process is called irreversible, if its reversed process is impossible (in nature).

According to this definition the reversibility or irreversibility of a process depends on the observer's experience. This is an awkward situation because experience of different observers may be different. Therefore we need a general criterion for describing the irreversibility of a process. Such a criterion is given by one of the different formulations of the second law which are discussed in the next section. Here we restrict ourselves to the phenomenological description of (many-body) systems, that means, we consider thermodynamics without a statistical or transporttheoretical background.

But before discussing the formal criteria of irreversibility we want to ask: Why is the diffusion into vacuum, considered above, an irreversible process? That means, why its reversed process cannot be observed in nature (but only in the backward running movie)? For producing the reversed process in nature we have to achieve a reversal of motion, that means, the moments of all the system's particles have to be reversed at a certain time. Such a preparation of a many-body system is not possible. Thus, suitable initial conditions cannot be installed in such a way, that the reversed process launches. Eigen calls this reason for irreversibility weak temporality [3]. Another reason for irreversibility, the strict temporality is discussed in Section 4.

\section{Clausius' inequality}

As mentioned in the introduction there are, in nature, no reversed processes of irreversible processes, because the initial conditions generating such processes cannot be prepared. This fact is expressed in thermodynamics by the second law, which is dressed in different formulations. For writing down a verbal formulation of the second law we need two concepts, the perpetuum mobile of second kind and the friction process. Both these concepts are now discussed.

A special perpetuum mobile of second kind is the following process: A ship is equipped by a drive machine, which absorbs energy from the sea by cooling it down. Although the energy conservation law is satisfied-the motive energy is absorbed from the sea-such a machine, called Kelvin machine, does not exist. It would be a perpetuum 
mobile of second kind, which absorbs heat from the sea, transferring it totally into work. But the reversed process does exist in nature: Work can be transferred totally into heat, what is denoted as a friction process. We now can give a verbal formulation of the second law:

2nd Law I : There is no perpetuum mobile of second kind, but friction processes exist.

The (possible) friction process as well as the non-existing (impossible) Kelvin process can be represented by thermodynamic diagrams [4] by which a lucid derivation of Clausius' inequality from the verbal formulation I of the second law can be achieved. This inequality which can be derived also by standard methods is the analytic formulation of the second law for closed systems ${ }^{2}$.

2nd Law II: All possible cyclic processes in closed systems satisfy Clausius' inequality

$$
+\oint \frac{\dot{Q}(t)}{T^{*}(t)} d t \leq 0
$$

As the derivation of this inequality from I shows $+\oint$ is the path integral along a cyclic process which at least contains one state of equilibrium [5], $\dot{Q}(t)$ is the heat exchange rate between the system and its environment at time $t$ and $T^{*}(t)$ is the absolute temperature of the environment which because of its reservoir properties is always presupposed to be in equilibrium. Here equilibrium is defined as follows:

Definition: Time independent states of isolated systems are called states of equilibrium. All equilibrium states span the equilibrium sub-space.

This definition as well as the path integral (1) demands that we choose a state space [6], what we will not discuss here in more detail.

A system in equilibrium is time-independent and isolated by definition. Hence we have $\dot{Q}(t)=0$, and the equality appears in Clausius' inequality for equilibrium states. Consider a trajectory only consisting of equilibrium states. This process does not exist in nature, because no possible process can only consist of equilibrium states: It would not proceed. But the equilibrium trajectories are of important significance: They are included in Clausius' inequality as boundary elements, and in contrast to possible processes they have, as equilibrium trajectories, no transit orientation. Therefore we define:

Definition: Trajectories in equilibrium sub-space are denoted as reversible processes.

The Kelvin process discussed above contradicts Clausius' inequality (1), because the heat exchange $\dot{Q}(t)$ absorbed by the Kelvin machine as well as the temperature $T^{*}(t)$ are positive at all times. We discussed already that the reversed diffusion process is impossible. Therefore it cannot satisfy Clausius' inequality what can be demonstrated as follows:

\footnotetext{
${ }^{2)}$ no material exchange between system and its environment
} 
First of all, we consider the diffusion process. Because it takes' place in an isolated system we have $\dot{Q}(t)=0$, and the internal energy $U(t)$ is a constant. To apply Clausius' inequality we have to complete the diffusion process reversibly to a cyclic process. For this purpose we introduce a piston by which the gas is compressed reversibly to its initial volume. Because the compression work is positive, and the final state of the compression is just the initial state of the diffusion process, we obtain a negative heat exchange according to the 1st law. Therefore, Clausius' inequality is satisfied for the irreversible diffusion process. For its reversed process the heat exchange has to be replaced by its negative, and consequently Clausius' inequality is violated.

We now discuss other formulations of the second law which follow from Clausius' inequality.

\section{Entropy production}

Clausius' inequality (1) is now transformed by adding the entropy production $\Sigma$ integrated along the cyclic process:

$$
+\oint\left[\frac{\dot{Q}(t)}{T^{*}(t)}+\Sigma(t)\right] d t=0, \quad+\oint \Sigma(t) d t \geq 0
$$

Thus (1) results in the following equivalent statement

2nd Law III: All possible processes in closed systems satisfy the dissipation inequality

$$
+\oint \sum d t \geq 0
$$

which does not determine the sign of $\Sigma$. The integrand of $(2)_{1}$ is called rate of entropy

$$
\dot{S}(t):=\frac{\dot{Q}(t)}{T^{*}(t)}+\sum(t)
$$

and from (4) and (2) we obtain the so-called embedding axiom [7]

$$
+\oint \dot{S}(t) d t=0
$$

In general the entropy rate (4) is not a time derivative of a state function because it depends on the controlling of the process by the temperature $T^{*}(t)$ of its environment. Often an entropy $S(t)$ can be introduced which is a functional of the process history ${ }^{3)}$ [8], i.e. the properties of the considered system depend on its past history. Examples for such dependence of the material properties on the past history of the process are hysteresis phenomena, e.g. in ferromagnets (cf. Fig. 1), or after-effects during the formation of materials, e.g. creeping of concrete.

\footnotetext{
3) A functional is a mapping which is defined on processes, i.e. the value of the functional depends on states in the past history of the system. In contrast a function is defined on the state space and not on trajectories in it.
} 
The global property (3) of the entropy production is too weak to determine its sign because we can not conclude from the sign of an integral to that of the integrand. Therefore we demand, that the entropy production satisfies the follwing extended, in time local inequalities:

2nd Law IV: The extended dissipation inequality is valid at each time $t$

$$
\begin{aligned}
& \Sigma(t)>0, \text { along irreversible processes, } \\
& \Sigma(t)=0, \text { along reversible processes. }
\end{aligned}
$$

The extension of the dissipation inequality (3) is achieved by introducing a strict positive entropy production for all non-equilibrium states instead of (3), whereas for all equilibrium states the entropy production vanishes.

By use of the extended dissipation inequality (6) we obtain from (4) another formulation of the second law for the rate of entropy:

2nd Law V: In closed systems the entropy rate is bounded from below

$$
\dot{S}(t) \geq \frac{\dot{Q}(t)}{T^{*}(t)}
$$

From (8) we obtain immediately the special formulation

2nd Law Va: In closed systems the rate of entropy is positive along irreversible adiabatic processes

$$
\dot{S}>0 \text {. }
$$

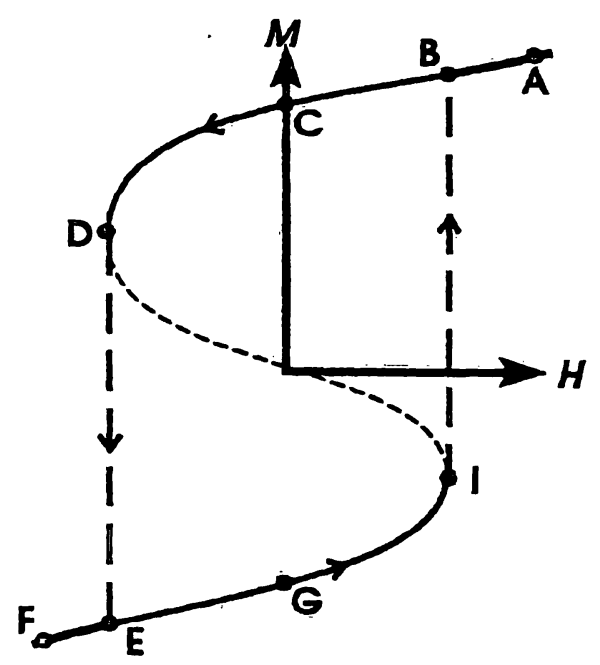

Fig. 1: Plot of a constitutive functional $M$ defined on state space $H$. The value of the functional is history dependent: Influenced by the history different values of $M$, here $C$ und $G$, belong to $M=0$. 


\section{Strict temporality}

Before exploiting the embedding axiom (5) we discuss a second reason for irreversibility considering the example of hysteresis. If constitutive properties have to be represented by functionals because they depend on the process history, there arises the possibility that bifurcations may appear (cf. Fig. 1). A system is in the state $A$ and runs via $B$ and $C$ to $D$. Here a jump of the constitutive property $M$ from $D$ to $E$ takes place, and the system arrives at the state $F$. We now assume that the time development of variable $H$ can be reversed. This assumption does not correspond to the reversal of motion, because the moments (velocities) of all particles are not reversed, but only the time derivatives of a special set of macroscopic variables. This is a realistic assumption, because such variables exist, e.g. the external magnetic field applied to a ferromagnetic system. After having reversed the time derivatives of these variables the system is running back to $E$. Coming from $F$ there is no reason in $E$ that the system jumps to $D$. But it runs through the states $G$ and $I$ ' on the 'lower' sheet of the material functional which is many-valued. Thus $G$ and $C$ belong to the same state $H=0, G$ on the lower sheet, $C$ on higher one. If in $I$ the value of $H$ becomes greater, the system jumps from the lower to the higher sheet to $B$. If $H$ increases, the system will reach its initial state $A$. The process through $A, \mathrm{~B}, C, \ldots, I, B, A$ is a hysteresis. Depending on the past history different values belong to the state $H$.

The bifurcations in $B$ and $E$ cause irreversibility: The system does not run backward, if $H$ is reversed, because in $E$ we have the 'correct' value of $H$, so that a jump to $D$ does not appear. The oriented jumps $D \rightarrow E$ and $I \rightarrow B$ are catastrophes in Thom's sense [9]. Eigen denotes the irreversibility caused by catastrophes as strict temporality of a process [3].

As discussed, we have irreversibility, if strict temporality appears. But beyond that, the processes are unpredictable, although they are deterministic. If we consider e.g. a system in the vicinity of the state $D$. It may jump to $E$ by thermal fluctuations, even if the external conditions are fixed. If this jump from $D$ to $E$ will really take place in the considered system, and how long we have to wait for it, is unpredictable and can only be calculated as a mean value over many systems. But if the system has jumped to $E$, it cannot go back to $D$ by fluctuations because of the irreversibility. Consequently, strict temporality is characterized by unpredictability and irreversibility as well.

The irreversibility of natural processes is often described by arrows of time. This diction means, that irreversible processes are directed in time. Depending on the considered phenomenon we distinguish between different arrows of time [10]: The thermodynamic arrow of time, the radiation arrow of time, the quantum-mechanical arrow of time, and the arrow of time of the cosmic space-time. All these arrows of time characterize different irreversible processes. In our context the question arises, if a thermodynamic arrow of time can be derived by the second law. From his nature the second law is a no-go theorem, i.e. according to formulation I it states, that Kelvin processes do not appear in nature, but their reversed processes are possible ones. The second law does not give any arguments for the impossibility of Kelvin processes. In contrast to this, weak and strict temporality make a proposition why a reversal of processes is impossible. Each temporality gives a different reason for irreversibility. Because the weak temporality represents a more technical statement about the 
preparation of initial conditions, we first of all suppose, that the strict temporality results in an explanation of irreversibility. But there are materials which do not show hysteresis properties, i.e. which do not obey strict temporality. Processes in such materials are in general also irreversible. This irreversibility, because not generated by strict temporality, is caused by the weak one. Consequently we obtain the following

Statement: The thermodynamic arrow of time is either caused by constitutive properties which generate bifurcations, or/and it arises by the impossbility to prepare arbitrary initial conditions.

\section{Entropy}

We now exploit the embedding axiom (5). For that purpose we consider a reversible cyclic process $\mathscr{K}$ which by definition takes a trajectory in the equilibrium sub-space. Hence (5) results in

$$
\mathscr{K} \oint \dot{S} d t=0, \quad \text { for all } \mathscr{K} \text { in the equilibrium sub-space. }
$$

Because (10) is valid for all cyclic processes in equilibrium sub-space, $\dot{S}$ is the time derivative of a function being unique up to a constant which we call equilibrium entropy $S^{e q}$. This argumentation is not applicable for cyclic processes out of equilibrium sub-space, because (5) as well as (2) are not valid for arbitrary cyclic processes, but only for those which contain at least one equilibrium state [5]. The equilibrium entropy does not depend on the process, therefore it is not a functional of the process history. Thus we have for the reversible process $\mathscr{C}$ between the equilibrium states $B(e q)$ and $A(e q)$

$$
\mathscr{C} \int_{B(e q)}^{A(e q)} \dot{S} d t=S_{A}^{e q}-S_{B}^{e q}
$$

We now consider an adiabatic irreversible process

$$
\mathscr{F}: A(e q) \rightarrow B(e q), \quad \dot{Q}(t)=0
$$

which launches from an equilibrium state $A$ and arrives at an equilibrium state in $B$. We now introduce an equilibrium trajectory (a reversible process) between $B$ and $A$

$$
\mathscr{R}: \mathrm{B}(e q) \rightarrow A(e q), \quad \Sigma(t)=0 .
$$

The convolution of both these processes results in a cyclic process for which (5) can be written down under consideration of (12) as

$$
0=+\oint \dot{S}(t) d t=\mathscr{F} \int \Sigma(t) d t+\mathscr{R} \int \dot{S} d t
$$

Consequently we obtain another formulation of the second law by use of (6) and (11):

J. Non-Equilib. Thermodyn. $\cdot 1998 \cdot$ Vol. $23 \cdot$ No. 1 
2nd Law VI: The equilibrium entropy increases, if an irreversible adiabatic process connects two equilibrium states $A(e q)$ and $B(e q)$ :

$$
S_{B}^{e q}>S_{A}^{e q}
$$

We now will derive a non-equilibrium entropy from (4). For that purpose we consider an irreversible process $\mathscr{T}$ in an isolated system

$$
\mathscr{T}: \mathrm{C} \rightarrow D(e q), \quad \dot{Q}=0,
$$

and (4) is integrated along $\mathscr{T}$

$$
\mathscr{T} \int_{C}^{D} \dot{S}(t) d t=\mathscr{T} \int_{C}^{D} \Sigma(t) d t=: S_{D}^{e q}-S_{C}
$$

By use of (6) we obtain another formulation of the second law:

2nd Law VII: The equilibrium entropy of an isolated system is always greater than its entropy in non-equilibrium

$$
S_{D}^{e q}>S_{C}
$$

It is remarkable that from (17) the existence of a non-equilibrium entropy follows, if the entropy production is history independent

$$
S_{C}=S_{D}^{e q}-\mathscr{T} \int_{C}^{D} \Sigma(t) d t
$$

and if along $\mathscr{T}$ no bifurcations appear, so that according to (16) from $C$ always $D(e q)$ uniquely follows in the isolated system.

\section{Field formulation}

Without any exception the seven discussed formulations of the second law refer to discrete systems. These are systems filling up a certain area in space which can be separated from its environment. The quantities describing the system belong to the system as a whole. These quantities as $\Sigma, \dot{S}, S^{e q}, S_{\mathrm{C}}$ are not fields, they do not depend on position, but belong to the entire discrete system.

If we want to describe a system in more detail as we can do by the concept of discrete system, we have to introduce the physical quantities as fields. Then a field formulation of thermodynamics results which contains the balance equations of mass, momentum, moment of momentum, total energy, internal energy and entropy, and additionally the constitutive equations. In our context with respect to the second law only the balance of entropy is of interest. It runs as follows:

$$
\partial_{t}(\rho s)+\nabla \cdot[\rho s v+\Phi]-\gamma=\sigma
$$


The fields appearing in (20) have the following meaning:

$$
\begin{aligned}
& \rho(x, t)=\text { mass density, } \\
& s(x, t)=\text { specific }^{4)} \text { entropy, } \\
& \boldsymbol{v}(\boldsymbol{x}, t)=\text { material velocity, } \\
& \boldsymbol{\Phi}(\boldsymbol{x}, t)=\text { conductive }^{5)} \text { entropy flux density, } \\
& \gamma(x, t)=\text { entropy supply }^{6}, \\
& \sigma(x, t)=\text { entropy production density }^{7)} .
\end{aligned}
$$

The partial time derivative is denoted by $\partial_{t}$, the derivative to position as $\nabla$. The second law in field formulation runs as follows:

2nd Law VIII: The field of the entropy production density of possible and reversible processes is non-negative for all times and positions:

$$
\sigma(x, t) \geq 0, \quad=\text { along reversible processes. }
$$

The entropy production of a discrete system $\mathscr{G}$ is determined by its density. By integration we obtain from (27) the inequality

$$
\Sigma(t)=\int_{\mathscr{S}} \sigma(x, t) d V \geq 0 .
$$

Consequently the formulation IV of the second law follows from VIII. This demonstrates that the different formulations of the second law are not independent of each other. The relations between them are discussed in the next section in more detail.

\section{Logical relations}

Up to here we have considered eight different, but not independent formulations of the second law. These eight formulations are of course not all possible ones: We mentioned already the saying that "there are more formulations of the second law than there are authors" [2]. Now we will discuss the logical relations between these eight formulations.

As already derived at the end of the last section we have

$$
\mathrm{VIII} \rightarrow \mathrm{IV},
$$

and in the second section before (1) we mentioned that

\footnotetext{
4) per mass

5) the velocity independent part

6) time rate of entropy by volume effects, e.g. absorption

7) time rate of entropy by irreversibility
}

J. Non-Equilib. Thermodyn. $1998 \cdot$ Vol. $23 \cdot$ No. 1 


$$
\mathrm{II} \leftrightarrow \mathrm{I}
$$

can be proved ${ }^{8)}$. Because (3) follows directly from (6) and (7) we have

$$
\text { IV } \rightarrow \text { III. }
$$

Analogously V (8) follows directly from IV by use of (4) and vice versa

$$
\mathrm{IV} \leftrightarrow \mathrm{V} \rightarrow \mathrm{Va},
$$

and just so from (17) and (18) we can read off the statement

$$
\text { IV } \rightarrow \text { VII. }
$$

Further we can conclude from the definition of the rate of entropy (4) and the embedding axiom (E-Ax) (5) by integration and inserting the two statements

$$
\mathrm{II} \wedge \mathrm{E}-\mathrm{Ax} \rightarrow \mathrm{III}, \quad \text { III } \wedge \mathrm{E}-\mathrm{Ax} \rightarrow \mathrm{II} .
$$

From (14) and (15) we can read off

$$
\mathrm{IV} \wedge \mathrm{E}-\mathrm{Ax} \rightarrow \mathrm{VI} \text {. }
$$

These seven statements (29) to (35) have now to be exploited.

First of all we see that the embedding axiom (5) is always a true statement because of its axiomatic character as a demand for the entropy rate: There is no entropy rate which does not satisfy (5). Consequently, E-Ax in (34) and (35) does not represent any constraint for the logical statements, and we obtain:

$$
\text { II } \rightarrow \text { III, III } \rightarrow \text { II, IV } \rightarrow \text { VI. }
$$

From the first two statements follows immediately

$$
\text { II } \leftrightarrow \text { III. }
$$

As a result we obtain the following diagram:

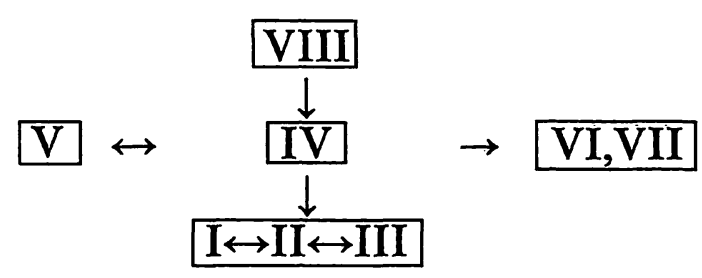

which we will discuss in the next section.

\section{Discussion}

The logical structure of the diagram below eq. (37) is as follows: The verbal formulation I, Clausius' inequality II, and the dissipation inequality III are in time integrated formulations of the 2 nd law which are equivalent to each other. They are more general

8) The proof is standard and can be extended also to negative absolute temperatures [4] 
than the in time local formulations which-as the conclusion arrows show-cannot be derived from the statements being global in time. From the extended dissipation inequality IV, criteria for the entropy rate $\mathrm{V}$ and the entropies in equilibrium and non-equilibrium VI and VII follow. The most special version is that of the field formulation VIII from which all other formulations can be derived.

Now the question arises, if by use of the more special formulations VIII and IV which are valid for less processes than III allows, processes are excluded which do not satisfy $\sigma \geq 0$ or $\Sigma \geq 0$ for all times at all positions, but which obey the integral criterion III. If fields are changing in time rapidly, the following example demonstrates, that the (in time local) entropy production or its density may be negative in short time intervals.

We consider a sedimentation process in a strong and homogeneous external acceleration field which is switched off in a very short time. Then by inertia the particles are moving for a short time against the gradient of mass concentration in a space which is free of external fields. In this time the entropy production is negative. Consequently, in time local formulations of the 2 nd law are true for sufficiently aged systems.

Even if we restrict ourselves to aged systems, the following question arises: What are the consequences of the extended formulation VIII of the 2nd law? Does it select the possible processes or the possible materials? This question cannot be answered by (27) because nothing is said about the interpretation of $\sigma<0$ : Are processes forbidden which do not fulfill VIII, or do only materials exist which satisfy VIII? First of all this question appears as unimportant, but for exploiting the dissipation inequality (27) in continuum thermodynamics one has to know the answer. This shows that the 2 nd law in its formulation VIII is incomplete and needs an amendment. This amendment runs as follows [11]:

Non-reversible direction axiom: Except in equilibria reversible process directions in state space do not exist.

By this axiom the question posed above can be answered: There are no materials violating VIII. Another result of the non-reversible direction axiom is that the entropy production density (27) is a state function on great state spaces [12].

\section{Summary}

There are different formulations of the 2 nd law which are not equivalent to each other. The verbal no-go formulation concerning the perpetuum mobile of second kind, Clausius' inequality, and the in time integrated entropy production inequality are the general formulations being equivalent to each other. They can be derived (but not vice versa) from the extended formulations, that are the two in time local dissipation inequalities of discrete systems and of cor tinuum thermodynamics. These in time local formulations are only true for sufficiently aged systems. Beyond that, they are incomplete because without an amendment one cannot decide, if these formulations are process-restrictive or material-restrictive. By adding this amendment-the nonreversible direction axiom-the second law in its local formulations turns out to be material-restrictive. 


\section{Acknowledgement}

I thank Dr. Gerald Paul, Vishay Company, D-95100 Selb, Germany, for continuous and generous support of our group.

\section{References}

[1] Muschik, W., Formulations of the second law-Recent developments. J. Phys. Chem. Solids, 49 (1988), 709-720.

[2] Hutter, K., The foundation of thermodynamics, its basic postulates and implications, Acta Mech., 27 (1977), 1-54.

[3] Eigen, M., Evolution und Zeitlichkeit, Die Żeit, Schriften der Carl Friedrich von Siemens Stiftung, Vol 6, p. 45, Oldenburg, 1983.

[4] Muschik, W., Thermodynamical algebra, second law, and Clausius' inequality at negative absolute temperatures, J. Non-Equilib. Thermodyn. 14 (1989), 173-198.

[5] Muschik, W., Aspects of Non-Equilibrium Thermodynamics, Sect. 5.4, World Scientific, Singapore, 1990.

[6] Muschik, W., Dominguez-Cascante, R., On extended thermodynamics of discrete systems, Physica A, 233 (1996), 523-550.

[7] Muschik, W., Fundamentals of dissipation inequalities, I. Discrete systems, J. Non-Equilib. Thermodyn. 4 (1979), 277-294.

[8] Muschik, W., Fundamentals of Non-Equilibrium Thermodynamics, in: Non-Equilibrium Thermodynamics with Application to Solids, Sect. 2, Ed. Muschik, W. Springer Wien, 1993.

[9] Thom, R., Structural Stability and Morphogenesis, Benjamin, London, 1975.

[10] Zeh, H. D., Die Physik der Zeitrichtung, Lecture Notes in Physics 200, Springer, 1984.

[11] Muschik, W., Ehrentraut, H., An amendment to the Second Law, J. Non-Equilib. Thermodyn. 21 (1996), 175-192.

[12] Muschik, W., Papenfuss, C., Ehrentraut, H., Concepts of Continuum Thermodynamics, 5 Lectures on Fundamentals, Methods, and Examples, Kielce University of Technology, 1996.

Paper received: 1997-9-16

Paper accepted: 1997-10-1

\section{Professor}

Dr. Wolfgang Muschik

Institut für Theoretische Physik

Technische Universität Berlin

Hardenbergstraße 36

D-10623 Berlin

Germany 